\title{
Speech processing for the hearing-impaired: successes, failures, and implications for speech mechanisms
}

\author{
Brian C.J. Moore * \\ Department of Experimental Psychology, University of Cambridge, Downing Street, Cambridge CB2 3EB, UK
}

\begin{abstract}
People with sensorineural hearing impairment typically have more difficulty than normally hearing people in understanding speech in the presence of background sounds. This paper starts by quantifying the magnitude of the problem in various listening situations and with various types of background sound. It then considers some of the factors that contribute to this difficulty, including: reduced audibility; reduced frequency selectivity; loudness recruitment; and regions in the cochlea which have no surviving inner hair cells and/or neurones (dead regions). Methods of compensating for the effects of some of these factors are described and evaluated. Signal-processing methods to compensate for the effects of reduced frequency selectivity using the output of a single microphone have had only limited success, although methods using multiple microphones have worked well. Amplitude compression can compensate for some of the effects of loudness recruitment, allowing speech to be understood over a wide range of sound levels. The exact form of the compression (fast-acting versus slow-acting, single-channel versus multiple channel) does not seem to be critical, suggesting that the relative loudness of different components of speech, and dynamic aspects of loudness perception do not need to be restored to "normal".
\end{abstract}

(c) 2002 Elsevier Science B.V. All rights reserved.

Keywords: Speech intelligibility; Hearing impairment; Effects of noise; Speech enhancement; Loudness recruitment

\section{Introduction}

The most common type of hearing loss is sensorineural hearing loss, which is typically associated with a dysfunction of the cochlea. In this article, I will focus on hearing loss that is assumed to be primarily cochlear in origin. It is well known that people with cochlear hearing impairment perform more poorly than normally hearing people when trying to understand speech, especially

\footnotetext{
${ }^{*}$ Tel.: +44-1223-333574; fax: +44-1223-333564.

E-mail address: bcjm@cus.cam.ac.uk (B.C.J. Moore).
}

when background noise is present. This paper considers the following questions:

(1) How much worse than normal are the hearing impaired at discriminating speech in noise?

(2) Why are the hearing impaired worse at discriminating speech in noise? I will consider both the role of reduced audibility and the role of supra-threshold "distortions" such as reduced frequency selectivity and loudness recruitment.

(3) What methods are available for compensating for the effects of reduced frequency selectivity and loudness recruitment, and how do these methods compare in effectiveness? 
(4) What implications do these findings have for mechanisms of speech perception?

\section{Difficulty in understanding speech in noise: How much worse are the hearing impaired?}

The issue considered first is: how much worse than normal are hearing-impaired people in their ability to understand speech in noise? This is often quantified by estimating the speech-to-noise ratio required to achieve a certain degree of intelligibility, such as $50 \%$ correct. This ratio is called the speech reception threshold (SRT) and it is usually expressed in $\mathrm{dB}$. The higher the SRT, the poorer is performance. For many of the common speech materials used, and especially for sentence lists (Bench and Bamford, 1979; Plomp and Mimpen, 1979; Nilsson et al., 1994), the percent correct varies quite rapidly with changes in speechto-noise ratio. For example, if the speech-to-noise ratio is set to a value giving about $50 \%$ correct, increasing the ratio by $1 \mathrm{~dB}$ typically gives an increase in percent correct of $7-19 \%$. Correspondingly, even small differences in SRT between normally hearing and hearing-impaired listeners indicate substantial differences in the ability to understand speech in noise.

Table 1 summarises the outcome of several studies. It shows the amount by which the SRT in noise is higher for people with cochlear hearing loss than for normally hearing people, in various listening situations. When the background is speech-shaped noise, with the same long-term average spectrum as the speech, and the speech and noise both come from the same direction (or are delivered monaurally or diotically via earphones) the deficit in SRT varies from about $2.5 \mathrm{~dB}$ for people with mild hearing losses caused by noise exposure or associated with ageing, to about $7 \mathrm{~dB}$ for people with moderate to severe losses caused by Ménière's syndrome or by unknown pathologies (Plomp, 1994). Providing frequency-selective amplification, via a hearing aid or by other means, reduces the deficit only slightly, typically by $1-2$ dB (Peters et al., 1998; Bentler and Duve, 2000).

The deficit in SRT is greater, typically 6-12 dB, when a fluctuating background noise or a single competing talker is used instead of a steady noise. Normally hearing people are able to take advantage of temporal and spectral "dips" in the interfering sound to achieve a much lower SRT than when steady background noise is used. People with cochlear hearing loss seem less able than normally hearing people to take advantage of the temporal and spectral dips (Duquesnoy, 1983; Festen and Plomp, 1990; Hygge et al., 1992; Baer and Moore, 1994; Moore et al., 1995; Peters et al., 1998). Amplification typically produces only a small improvement in the SRT in fluctuating background sounds (Peters et al., 1998; Moore et al., 1999).

Finally, people with cochlear hearing loss are less able than normally hearing people to take advantage of spatial separation of the target speech and the interfering sound(s). When the background sound is a single talker spatially separated from the target speech, the deficit in SRT is 12-19 dB (Duquesnoy, 1983). This represents a very substantial problem. However, the majority of laboratory experiments show a less severe problem, as they have used as a background sound steady speech-shaped noise coming from the same direction as the target speech (or presented to the same ear via earphones).

Table 1

Typical amounts by which the SRT in background sounds is greater for hearing-impaired than for normally hearing listeners, for various types of background sounds and listening situations

\begin{tabular}{llr}
\hline Type of background & Listening situation & Deficit in SRT (dB) \\
\hline Speech-shaped noise & Speech + background in front, unaided & $2.5-7$ \\
Speech-shaped noise & Speech + background in front, aided & $2-6$ \\
Single talker & Speech + background in front, unaided & $6-12$ \\
Single talker & Speech + background in front, aided & $4-10$ \\
Single talker & Speech + background spatially separated & $12-19$ \\
\hline
\end{tabular}




\section{Causes of difficulty in understanding speech in noise}

\subsection{Reduced audibility}

There is no doubt that audibility is crucial for speech intelligibility; if part of the speech spectrum is below the absolute threshold or is masked by background sound, then information is lost, and intelligibility will suffer to some extent. Loss of audibility may be especially important when the target speech and background are spatially separated, as is typically the case in everyday life. In this situation, "head shadow" effects often lead to an improved speech-to-background ratio at one ear, and these effects are greatest at high frequencies. A loss of ability to hear high frequencies may drastically reduce the ability to take advantage of head shadow effects (Bronkhorst and Plomp, 1989).

The articulation index (AI) provides a way of quantifying the effect of audibility on speech intelligibility (French and Steinberg, 1947; ANSI, 1969). In recent work, the term speech intelligibility index has been used instead of AI, but the underlying concepts are similar (ANSI, 1997). The AI is based on the assumption that speech intelligibility is uniquely related to a quantity that, for a normally hearing person, can be calculated from the long-term average spectra of the speech and background sound reaching the ear of the listener. The frequency range from about 200 to $9000 \mathrm{~Hz}$, which is the range most important for intelligibility, is divided into a number of bands. It is assumed that each band makes a certain contribution to speech intelligibility. That contribution is determined by the audibility of the speech in that band and by the relative importance of that band for intelligibility. The overall intelligibility is assumed to be related to a simple sum of the contributions from each band. Note that the version of the AI described by Fletcher (1953) is considerably more complex than this, and takes into account factors other than audibility. However, in what follows, I consider only the simpler audibility-based versions of the AI.

Several researchers have examined the question of whether the AI can be used to predict speech intelligibility for hearing-impaired listeners. While some have reported accurate predictions using the AI (Aniansson, 1974; Lee and Humes, 1993), most studies have shown that speech intelligibility is worse than would be predicted by the AI (Fletcher, 1952; Dugal et al., 1978; Pavlovic, 1984; Pavlovic et al., 1986; Smoorenburg, 1992; Ching et al., 1998; Hogan and Turner, 1998), especially for listeners with moderate or severe losses. Thus, factors other than audibility must contribute to the difficulties experienced by the hearing impaired. Some possible factors are considered next.

\subsection{Reduced frequency selectivity}

Frequency selectivity refers to the ability to resolve the spectral components in complex sounds. It is often characterised by assuming that the auditory system contains an array of bandpass filters-the auditory filters (Fletcher, 1940; Moore, 1997). The output of the filters, plotted as a function of centre frequency is called the excitation pattern, and it resembles a blurred version of the spectrum of the input signal (Moore, 1997). People with cochlear hearing loss usually have auditory filters that are broader than normal (Pick et al., 1977; Glasberg and Moore, 1986; Tyler, 1986). This means that their ability to determine the spectral shapes of speech sounds, and to separate components of speech from background noise, is reduced.

It is difficult to prove that reduced frequency selectivity is a cause of difficulty in understanding speech in noise (for a review, see Moore (1998)). However, simulations of the effect of reduced frequency selectivity, implemented by "smearing" of the short-term spectrum, strongly suggest that it is a contributing factor (ter Keurs et al., 1992, 1993; Baer and Moore, 1993, 1994; Nejime and Moore, 1997).

One mechanism by which impaired frequency selectivity could affect the identification of speech in noise involves the perception of spectral shape. Broader auditory filters produce a more highly smoothed representation of the spectrum (the excitation pattern) than normal auditory filters. If spectral features are not sufficiently prominent, they may be smoothed to such an extent that they 
become imperceptible. Adding a noise background to speech fills in the valleys between the spectral peaks and thus reduces their prominence, exacerbating the problem of perceiving them for people with broadened auditory filters.

A second possible effect of reduced frequency selectivity on speech perception in noise is connected with the temporal patterns at the outputs of individual auditory filters. The perceived frequency of a given formant and/or the fundamental frequency of voicing may be partly determined by the time pattern at the outputs of the auditory filters tuned close to the formant frequency (Rosen and Fourcin, 1986; Young and Sachs, 1979; Miller et al., 1997). Background noise disturbs this time pattern, which may lead to reduced accuracy in determining these frequencies. This effect would be greater in people with reduced frequency selectivity, since broader filters generally pass more background noise.

\subsection{Loudness recruitment}

Most people with cochlear hearing loss show a phenomenon called loudness recruitment (Fowler, 1936; Steinberg and Gardner, 1937). The absolute threshold is higher than normal, but when a sound is increased in level above the elevated absolute threshold, the rate of growth of loudness level with increasing sound level is greater than normal. When the level is sufficiently high, usually around 90-100 dB SPL, the loudness reaches its normal value; the sound appears as loud to the person with impaired hearing as it would to a normally hearing person. With further increases in sound level above 90-100 dB SPL, the loudness grows in an almost normal manner.

Loudness recruitment may affect speech intelligibility in several ways. Firstly, it is associated with a reduced dynamic range (the range between the absolute threshold and the highest comfortable level). This may adversely affect the ability to "listen in the dips" of a fluctuating background sound, such as a single talker. If the peaks in the background are amplified to the highest comfortable level, the level of target speech in the dips may be close to or below the absolute threshold. Secondly, loudness recruitment leads to a distorted loudness relationship among the components of speech sounds; the relative loudness of the components is different from normal. Finally, loudness recruitment leads to a distorted perception of amplitude modulation; the modulation depth is perceived as greater than normal (Moore et al., 1996). It has been argued by some researchers that the pattern of amplitude modulation of speech is important for intelligibility (Plomp, 1988; Shannon et al., 1995). If this is the case, then altered perception of the modulation patterns might be expected to lead to poorer speech intelligibility. However, evidence presented later in this paper suggests that this is not the case.

\subsection{Dead regions}

Dead regions are regions in the cochlea where there are no surviving inner hair cells and/or neurones, and hence there is no transduction of basilar membrane vibration (Moore and Glasberg, 1997; Moore et al., 2000; Moore, 2001). A dead region can be characterised in terms of the characteristic frequencies of the IHCs or neurones bordering that region. Dead regions can be diagnosed using by measuring psychophysical tuning curves (PTCs, see Thornton and Abbas, 1980; Florentine and Houtsma, 1983; Turner et al., 1983; Moore and Alcántara, 2001). A simpler test for use in the clinic has been described by Moore et al. (2000). This test involves measuring the detection threshold for pure tones in "threshold equalising noise" (TEN), so the test is called the "TEN test".

There are several theoretical reasons why people with dead regions might extract little or no information from frequency components of speech that fall within a dead region, even when those components are amplified sufficiently to make them audible. These reasons include:

(1) The frequency components are received through the "wrong" place in the cochlea. For example, if there is a low-frequency dead region, amplified low-frequency components will be detected and analysed via the frequency channels that are tuned to higher frequencies. This mismatch between frequency and place may lead to difficulty in interpreting the infor- 
mation derived from the low frequencies. There is some evidence supporting this idea from studies involving the simulation of hearing loss and/or of cochlear implant signal processing (Shannon et al., 1998). However, extended learning with "re-mapped" stimuli may partially compensate for this problem (Rosen et al., 1999).

(2) If the components falling in the dead region are amplified sufficiently to make them audible, they will be detected and analysed via the same neural channels that are used for other frequencies, and this may impair the analysis of those other frequencies. For example, if there is a low-frequency dead region, the amplified low-frequency components will be detected and analysed through the same neural channels as are used for the medium/high frequencies. Since speech is a broadband signal, usually containing components covering a wide frequency range, this may lead to some form of "information overload" in those channels.

(3) Information in speech, such as information about formant frequencies, may partly be coded in the time patterns of the neural impulses (phase locking). The analysis of temporal information may normally be done on a place-specific basis. For example, the neural machinery required to "decode" temporal information about frequencies around $1000 \mathrm{~Hz}$ may be restricted to neural channels tuned close to $1000 \mathrm{~Hz}$ (Loeb et al., 1983; Srulovicz and Goldstein, 1983). When there is a mismatch between the frequencies of the speech components and the place where they are detected, the temporal decoding mechanisms required to analyse those speech components may not operate effectively. The relative importance of these three factors is not known; all may be important to some extent.

Some of the effects of dead regions on speech intelligibility have been reviewed by Moore (2001). Here, I describe briefly a recent study of Vickers et al. (2001). Both PTCs and the TEN test were used to detect and define the limits of any dead regions. All subjects had high-frequency hearing loss, but some had high-frequency dead regions and some did not; generally, the subjects with dead regions had more severe high-frequency hearing losses than those without dead regions. The speech stimuli were vowel-consonant-vowel (VCV) nonsense syllables, using one of three vowels (/i/, /a/ and $/ \mathrm{u} /$ ) and 21 different consonants. In a baseline condition, subjects were tested using broadband stimuli with a nominal input level of $65 \mathrm{~dB}$ SPL. Prior to presentation via Sennheiser HD580 earphones, the stimuli were subjected to the frequency-dependent amplification prescribed by the "Cambridge" formula (Moore and Glasberg, 1998). The goal of the amplification was to restore audibility as far as possible, while avoiding excessive loudness. The stimuli for all other conditions were initially subjected to this same frequencydependent amplification. Then, the speech was lowpass filtered with various cutoff frequencies.

For subjects without dead regions, performance generally improved progressively with increasing cutoff frequency. An example is shown in the upper panel of Fig. 1. This indicates that they were able to make use of high-frequency information. For subjects with dead regions, two patterns of performance were observed. For some subjects, performance initially improved with increasing cutoff frequency and then reached an asymptote (Fig. 1, middle). This indicates that they were not able to make use of high-frequency information. For other subjects, performance initially improved with increasing cutoff frequency, and then worsened with further increases (Fig. 1, bottom). This indicates that amplification of high frequencies impaired performance.

It is noteworthy that, for subjects who showed an "optimum" cutoff frequency, the best performance was achieved when that cutoff frequency was $50-100 \%$ above the estimated edge frequency of the dead region. For subjects whose performance reached an asymptote, the asymptote was reached for a cutoff frequency about $70 \%$ above the estimated edge frequency of the dead region. Statistical analyses showed there was significant benefit from amplifying frequencies up to $70 \%$ above the estimated edge frequencies of the dead regions. Thus, hearing-impaired subjects are able to make some use of frequency components of 


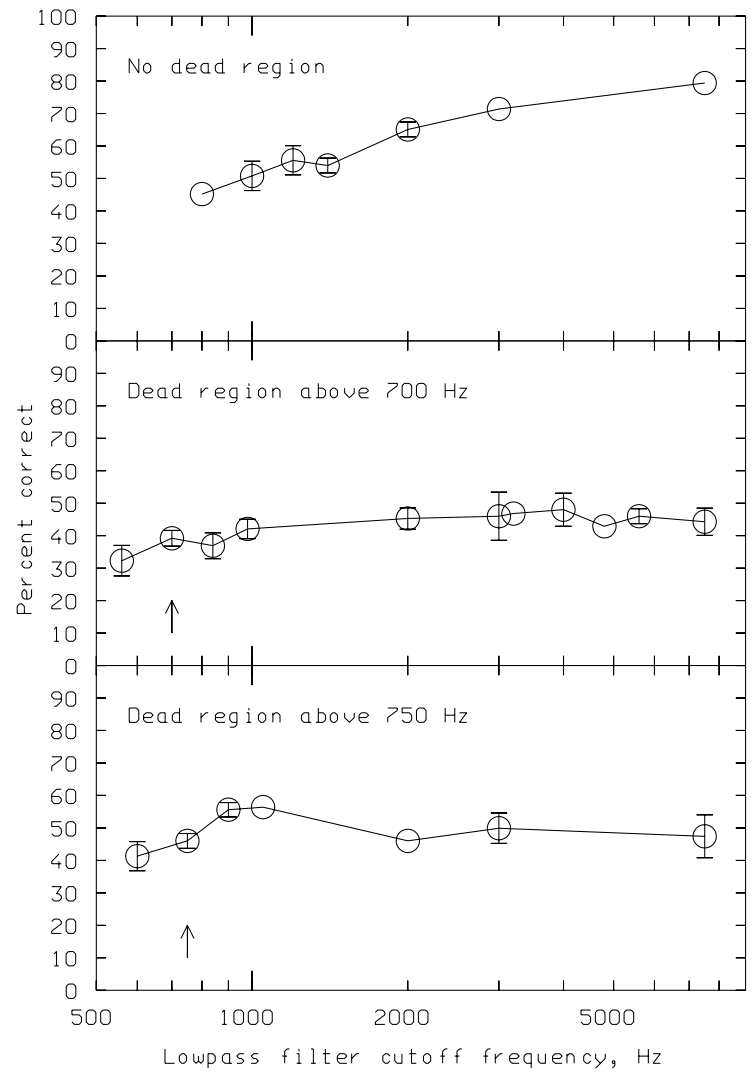

Fig. 1. Percent correct scores in identifying VCV syllables for three hearing-impaired subjects, one without (top) and two with (middle, bottom) a dead region. Scores are plotted as a function of the cut-off frequency of a lowpass filter. Prior to lowpass filtering, stimuli were given the frequency-gain characteristic prescribed by the Cambridge formula (Moore and Glasberg, 1998). Error bars indicate \pm one standard deviation across test sessions. Data are taken from (Vickers et al., 2001).

speech falling in a dead region, but only for frequencies that lie fairly close to the boundary of the dead region.

\section{Compensation for suprathreshold effects of hear- ing loss}

\subsection{Reduced frequency selectivity}

Many researchers have attempted to process speech in background noise to compensate for the effects of reduced frequency selectivity. For a re- view, see Moore (1995). Several methods are based on processing the signal picked up by a single microphone. One such method involves enhancement of the short-term spectrum; the contrast between spectral peaks and valleys is increased. This might be beneficial either because it reduces the level of the noise that fills the spectral valleys between formants, or because it makes the excitation pattern in an impaired ear more like that evoked in a normal ear by unprocessed stimuli. In practice, it is impossible to restore the short-term excitation pattern to normal, but an approximation to this can be achieved by rather extreme processing of the stimuli, replacing the most prominent spectral speaks by a small number of sinusoids. Such extreme processing has not been shown to enhance speech intelligibility (Kates, 1994; Baer and Moore, 1997).

Some other studies, using moderate amounts of spectral enhancement, have been shown to produce moderate improvements in speech intelligibility and in subjective ratings of speech quality (Simpson et al., 1990; Baer et al., 1993), although improvements have not always been found (Franck et al., 1999). So far, spectral enhancement has not been evaluated in real-time wearable devices, although a crude form of spectral enhancement has been implemented in a commercial digital hearing aid. It remains to be seen, therefore, whether greater benefits would be found with prolonged experience of spectrally enhanced stimuli.

Many other researchers have attempted to reduce the deleterious effects of background noise by using various forms of digital signal processing, such as spectral subtraction (Lim, 1983; Elberling et al., 1993). Also, many current hearing aids incorporate noise reduction systems where the input signal is filtered into several frequency bands, and the level is reduced in bands that are dominated by noise; the signal-to-noise ratio in each band is usually estimated from the amount or pattern of envelope modulation at the output of that band. On the whole, such schemes have produced little or no benefit for speech intelligibility, although they can improve listening comfort (Kuk et al., 1990; Tyler and Kuk, 1990; Alcántara et al., in press). For a review, see Dillon and Lovegrove (1993). 
A common problem for noise reduction schemes based on the output of a single microphone is the need to estimate the spectrum of the background noise. For schemes such as spectral subtraction, this is done using some form of "speech-pause detector", which attempts to discriminate time intervals containing background alone from time intervals containing signalplus-background. The background-alone intervals are used to estimate the spectrum of the background. It has proved to be extremely difficult to design robust and error-free speech-pause detectors. It appears that computational methods for segregating speech from background sounds are not nearly as good as those resulting from several million years of evolution.

The only signal processing schemes that have been clearly demonstrated to improve speech intelligibility are those based on the use of multiple microphones to create a highly directional characteristic (Soede et al., 1993; Ricketts and Mueller, 1999). Such systems are now widely used in hearing aids, and they can typically give an improvement in the SRT of 4-5 dB when the target speech comes from the front and there are several spatially distributed background sounds.

\subsection{Loudness recruitment}

Some researchers (e.g. Villchur, 1973; Hohmann and Kollmeier, 1995) have argued that a hearing aid should be designed to restore loudness perception to normal. If speech perception depended on the relative loudness of the different frequency components, or on normal perception of loudness fluctuations over time, then this argument would be reasonable. However, it is not clear that this is the case. Restoration of loudness perception to normal would require fast-acting multi-channel compression. The compression needs to be fast to restore the perception of amplitude modulation to normal (Moore et al., 1996), and it needs to be applied independently in multiple channels to allow for variations in the amount of loudness recruitment with frequency. Essentially, fast-acting multi-channel compression can be thought of as mimicking the action of the "active" mechanism which operates in normal ears and which is often adversely affected by hearing impairment (Moore, 1998).

Other researchers (e.g. Plomp, 1988; Shannon et al., 1995; Greenberg and Arai, 1998) have argued that the amplitude modulation patterns in different frequency bands are critical for speech intelligibility. According to these researchers, it is important to preserve the amount of modulation; fast compression reduces the effective modulation depth (Stone and Moore, 1992), and this may impair intelligibility. On the other hand, restoration of the perception of loudness fluctuations to normal should not be important. Therefore slow compression should lead to higher intelligibility than fast compression.

The experiment described next provides a test of which of these viewpoints is more nearly correct. Stone et al. (1999) compared the effectiveness of several different forms of compression, using experimental digital hearing aids. The aids were physically identical, but were programmed in different ways to implement the compression systems. Two of the systems will be considered here:

(1) A fast-acting four-channel system. This reduced the effective modulation depth independently in different frequency bands, and made the loudness perception (including the perception of fluctuating sounds) closer to normal.

(2) A slow-acting single-channel system, with appropriate frequency-response shaping. This kept the overall level of speech comfortable, but did not affect the envelope modulation of the speech. It did not even come close to restoring loudness perception to normal.

Eight subjects with moderate to severe cochlear hearing loss were tested in a counter-balanced design. Subjects had at least two weeks experience with each system in everyday life before evaluation using the "Abbreviated profile of hearing aid benefit" (APHAB) (Cox and Alexander, 1995). This test is based on a questionnaire which requires subjects to rate how often they have problems in specific situations, such as "Unexpected sounds, like a smoke detector or alarm bell are uncomfortable" or "When I am having a quiet conversation with a friend, I have difficulty understanding". 
Response alternatives range from "Always (99\%)" to "Never (1\%). The results are grouped into four sub-scales: ease of communication (EC), understanding in reverberant environments (RV), understanding in background noise (BN) and aversiveness of sounds (AV). Subjects were also evaluated using measures of speech intelligibility in quiet (word lists at 50 and $80 \mathrm{~dB}$ SPL) (Boothroyd, 1968) and noise (sentence lists in speech-shaped noise, or that same noise amplitude modulated with the envelope of speech from a single talker) (MacLeod and Summerfield, 1990).

The results showed that speech intelligibility in quiet and in background noise was similar for the two systems; both systems led to rather good performance, the SRTs in noise being generally negative (but not quite as low as for normally hearing people listening unaided). Subjective ratings of problems with speech communication (the $\mathrm{EC}, \mathrm{RV}$ and BN scales of the APHAB test) showed no significant difference between the two systems. This experiment, and others, leads to the following conclusions:

(1) Restoring loudness perception to normal is not necessary. This is demonstrated by the relatively good performance achieved with the slow-acting single-channel compression system. Good performance with slow-acting systems has also been found in other studies (Moore and Glasberg, 1988; Moore et al., 1991).

(2) Compression of modulations in speech is not necessary. Again, this is demonstrated by the relatively good performance achieved with the slow-acting single-channel compression system, which did not affect the modulation depth of the speech for modulation rates above about $1 \mathrm{~Hz}$.

(3) Compression of modulations in speech is not harmful, provided the amount of compression is moderate. This is demonstrated by the good results achieved using the multi-channel fastacting system. Other studies have also demonstrated good performance (better than for linear amplification) for multi-channel fastacting systems (Moore et al., 1992; Yund and Buckles, 1995a,b; Moore et al., 1999). Further- more, studies using normally hearing listeners have shown that multi-channel fast-acting compression of a moderate amount does not impair speech intelligibility (Noordhoek and Drullman, 1997; van Buuren et al., 1999).

The main benefit of compression is that it makes speech audible while maintaining listening comfort. The exact form of the compression does not appear to be critical, provided that it does not introduce marked distortion of the temporal envelope of the speech.

\section{Conclusions}

People with sensorineural hearing impairment perform more poorly than normally hearing people when trying to understand speech in the presence of background sounds. The deficit (relative to normal) is particularly large when the background is fluctuating and when the target speech and background are spatially separated. The deficit can be attributed to several factors: (1) reduced audibility, whereby part of the speech spectrum becomes inaudible; (2) reduced frequency selectivity, which contributes to difficulty in discriminating spectral shape and in separating speech and background frequency components; (3) loudness recruitment, which reduces the available dynamic range and distorts loudness relationships among components of speech; (4) dead regions in the cochlea, which prevent transduction of information at certain places on the basilar membrane.

Frequency-selective amplification can partly overcome the effects of reduced audibility. Attempts to compensate for the effects of reduced frequency selectivity, using the signal picked up by a single microphone, have had little or no success, although the use of multiple microphones to create a highly directional characteristic has given clear benefits. Several different forms of automatic gain control (compression) have been shown to be effective in compensating for the effects of loudness recruitment. It does not seem to be critical to restore loudness perception to normal. At present there is no effective method of compensating for the effects of dead regions (except with cochlear 
implants), but there is some benefit from amplifying frequency components that fall a little inside a dead region.

\section{Acknowledgements}

The work of the author's research group was supported by the MRC (UK), with additional support from the RNID, Defeating Deafness, GNResound, Starkey and Phonak. I thank an anonymous reviewer for helpful comments.

\section{References}

Alcántara, J.I., Moore, B.C.J., Kühnel, V., Launer, S. Evaluation of the noise reduction system in a commercial digital hearing aid. Int. J. Audiol. 41 (in press).

Aniansson, G., 1974. Methods for assessing high-frequency hearing loss in everyday situations. Acta Otolaryngol. Suppl. 320, 1-50.

ANSI, 1969. ANSI S3.5. Methods for the calculation of the articulation index. American National Standards Institute, New York.

ANSI, 1997. ANSI S3.5-1997, Methods for the calculation of the speech intelligibility index. American National Standards Institute, New York.

Baer, T., Moore, B.C.J., 1993. Effects of spectral smearing on the intelligibility of sentences in the presence of noise. J. Acoust. Soc. Am. 94, 1229-1241.

Baer, T., Moore, B.C.J., 1994. Effects of spectral smearing on the intelligibility of sentences in the presence of interfering speech. J. Acoust. Soc. Am. 95, 2277-2280.

Baer, T., Moore, B.C.J., 1997. Evaluation of a scheme to compensate for reduced frequency selectivity in hearingimpaired subjects. In: Jesteadt, W. (Ed.), Modeling Sensorineural Hearing Loss. Erlbaum, New Jersey.

Baer, T., Moore, B.C.J., Gatehouse, S., 1993. Spectral contrast enhancement of speech in noise for listeners with sensorineural hearing impairment: effects on intelligibility, quality and response times. J. Rehab. Res. Devel. 30, 49-72.

Bench, J., Bamford, J., 1979. Speech-Hearing Tests and the Spoken Language of Hearing-Impaired Children. Academic, London.

Bentler, R.A., Duve, M.R., 2000. Comparison of hearing aids over the 20th century. Ear Hear. 21, 625-639.

Boothroyd, A., 1968. Developments in speech audiometry. Sound 2, 3-10.

Bronkhorst, A.W., Plomp, R., 1989. Binaural speech intelligibility in noise for hearing-impaired listeners. J. Acoust. Soc. Am. 86, 1374-1383.

Ching, T., Dillon, H., Byrne, D., 1998. Speech recognition of hearing-impaired listeners: Predictions from audibility and the limited role of high-frequency amplification. J. Acoust. Soc. Am. 103, 1128-1140.

Cox, R.M., Alexander, G.C., 1995. The abbreviated profile of hearing aid benefit. Ear Hear. 16, 176-186.

Dillon, H., Lovegrove, R., 1993. Single-microphone noise reduction systems for hearing aids: A review and an evaluation. In: Studebaker, G.A., Hochberg, I. (Eds.), Acoustical Factors Affecting Hearing Aid Performance. Allyn and Bacon, Boston.

Dugal, R., Braida, L.D., Durlach, N.I., 1978. Implications of previous research for the selection of frequency-gain characteristics. In: Studebaker, G.A., Hochberg, I. (Eds.), Acoustical Factors Affecting Hearing Aid Performance. University Park Press, Baltimore.

Duquesnoy, A.J., 1983. Effect of a single interfering noise or speech source on the binaural sentence intelligibility of aged persons. J. Acoust. Soc. Am. 74, 739-743.

Elberling, C., Ludvigsen, C., Keidser, G., 1993. The design and testing of a noise reduction algorithm based on spectral subtraction. Scand. Audiol. Suppl. 38, 39-49.

Festen, J.M., Plomp, R., 1990. Effects of fluctuating noise and interfering speech on the speech-reception threshold for impaired and normal hearing. J. Acoust. Soc. Am. 88, 1725-1736.

Fletcher, H., 1940. Auditory patterns. Rev. Mod. Phys. 12, 47-65.

Fletcher, H., 1952. The perception of sounds by deafened persons. J. Acoust. Soc. Am. 24, 490-497.

Fletcher, H., 1953. Speech and hearing in Communication. Van Nostrand, New York.

Florentine, M., Houtsma, A.J.M., 1983. Tuning curves and pitch matches in a listener with a unilateral, low-frequency hearing loss. J. Acoust. Soc. Am. 73, 961-965.

Fowler, E.P., 1936. A method for the early detection of otosclerosis. Arch. Otolaryngol. 24, 731-741.

Franck, B.A.M., Sidonne, C., van Kreveld-Bos, G.M., Dreschler, W.A., Verschuure, H., 1999. Evaluation of spectral enhancement in hearing aids, combined with phonemic compression. J. Acoust. Soc. Am. 106, 1452-1464.

French, N.R., Steinberg, J.C., 1947. Factors governing the intelligibility of speech sounds. J. Acoust. Soc. Am. 19, 90 119.

Glasberg, B.R., Moore, B.C.J., 1986. Auditory filter shapes in subjects with unilateral and bilateral cochlear impairments. J. Acoust. Soc. Am. 79, 1020-1033.

Greenberg, S., Arai, T., 1998. Speech intelligibility is highly tolerant of cross-channel spectral asynchrony. J. Acoust. Soc. Am. 103, 3057.

Hogan, C.A., Turner, C.W., 1998. High-frequency audibility: Benefits for hearing-impaired listeners. J. Acoust. Soc. Am. 104, 432-441.

Hohmann, V., Kollmeier, B., 1995. The effect of multichannel dynamic compression on speech intelligibility. J. Acoust. Soc. Am. 97, 1191-1195.

Hygge, S., Rönnberg, J., Larsby, B., Arlinger, S., 1992. Normal-hearing and hearing-impaired subjects' ability to just follow conversation in competing speech, reversed 
speech, and noise backgrounds. J. Speech Hear. Res. 35, 208-215.

Kates, J.M., 1994. Speech enhancement based on a sinusoidal model. J. Speech Hear. Res. 37, 449-464.

Kuk, F.K., Tyler, R.S., Mims, L., 1990. Subjective ratings of noise-reduction hearing aids. Scand. Audiol. 19, 237-244.

Lee, L.W., Humes, L.E., 1993. Evaluating a speech-reception threshold model for hearing-impaired listeners. J. Acoust. Soc. Am. 93, 2879-2885.

Lim, J.S., 1983. Speech Enhancement. Prentice Hall, New Jersey.

Loeb, G.E., White, M.W., Merzenich, M.M., 1983. Spatial cross correlation: A proposed mechanism for acoustic pitch perception. Biol. Cybern. 47, 149-163.

MacLeod, A., Summerfield, Q., 1990. A procedure for measuring auditory and audio-visual speech-reception thresholds for sentences in noise: rationale, evaluation, and recommendations for use. Brit. J. Audiol. 24, 29-43.

Miller, R.L., Schilling, J.R., Franck, K.R., Young, E.D., 1997. Effects of acoustic trauma on the representation of the vowel $/ \varepsilon /$ in cat auditory nerve fibers. J. Acoust. Soc. Am. 101, 3602-3616.

Moore, B.C.J., 1995. Perceptual Consequences of Cochlear Damage. Oxford University Press, Oxford.

Moore, B.C.J., 1997. An Introduction to the Psychology of Hearing, fourth ed Academic, San Diego.

Moore, B.C.J., 1998. Cochlear hearing loss. Whurr, London.

Moore, B.C.J., 2001. Dead regions in the cochlea: Diagnosis, perceptual consequences, and implications for the fitting of hearing aids. Trends Amplif. 5, 1-34.

Moore, B.C.J., Alcántara, J.I., 2001. The use of psychophysical tuning curves to explore dead regions in the cochlea. Ear Hear. 22, 268-278.

Moore, B.C.J., Glasberg, B.R., 1988. A comparison of four methods of implementing automatic gain control (AGC) in hearing aids. Br. J. Audiol. 22, 93-104.

Moore, B.C.J., Glasberg, B.R., 1997. A model of loudness perception applied to cochlear hearing loss. Audit. Neurosci. 3, 289-311.

Moore, B.C.J., Glasberg, B.R., 1998. Use of a loudness model for hearing aid fitting. I. Linear hearing aids. Br. J. Audiol. 32, 317-335.

Moore, B.C.J., Glasberg, B.R., Stone, M.A., 1991. Optimization of a slow-acting automatic gain control system for use in hearing aids. Br. J. Audiol. 25, 171-182.

Moore, B.C.J., Glasberg, B.R., Vickers, D.A., 1995. Simulation of the effects of loudness recruitment on the intelligibility of speech in noise. Br. J. Audiol. 29, 131-143.

Moore, B.C.J., Huss, M., Vickers, D.A., Glasberg, B.R., Alcántara, J.I., 2000. A test for the diagnosis of dead regions in the cochlea. Br. J. Audiol. 34, 205-224.

Moore, B.C.J., Johnson, J.S., Clark, T.M., Pluvinage, V., 1992. Evaluation of a dual-channel full dynamic range compression system for people with sensorineural hearing loss. Ear Hear. 13, 349-370.

Moore, B.C.J., Peters, R.W., Stone, M.A., 1999. Benefits of linear amplification and multi-channel compression for speech comprehension in backgrounds with spectral and temporal dips. J. Acoust. Soc. Am. 105, 400-411.

Moore, B.C.J., Wojtczak, M., Vickers, D.A., 1996. Effect of loudness recruitment on the perception of amplitude modulation. J. Acoust. Soc. Am. 100, 481-489.

Nejime, Y., Moore, B.C.J., 1997. Simulation of the effect of threshold elevation and loudness recruitment combined with reduced frequency selectivity on the intelligibility of speech in noise. J. Acoust. Soc. Am. 102, 603-615.

Nilsson, M., Soli, S.D., Sullivan, J.A., 1994. Development of the Hearing in Noise Test for the measurement of speech reception thresholds in quiet and in noise. J. Acoust. Soc. Am. 95, 1085-1099.

Noordhoek, I.M., Drullman, R., 1997. Effect of reducing temporal intensity modulations on sentence intelligibility. J. Acoust. Soc. Am. 101, 498-502.

Pavlovic, C.V., 1984. Use of the articulation index for assessing residual auditory function in listeners with sensorineural hearing impairment. J. Acoust. Soc. Am. 75, 1253-1258.

Pavlovic, C., Studebaker, G., Sherbecoe, R., 1986. An articulation index based procedure for predicting the speech recognition performance of hearing-impaired individuals. J. Acoust. Soc. Am. 80, 50-57.

Peters, R.W., Moore, B.C.J., Baer, T., 1998. Speech reception thresholds in noise with and without spectral and temporal dips for hearing-impaired and normally hearing people. J. Acoust. Soc. Am. 103, 577-587.

Pick, G., Evans, E.F., Wilson, J.P., 1977. Frequency resolution in patients with hearing loss of cochlear origin. In: Evans, E.F., Wilson, J.P. (Eds.), Psychophysics and Physiology of Hearing. Academic Press, London.

Plomp, R., 1988. The negative effect of amplitude compression in multichannel hearing aids in the light of the modulationtransfer function. J. Acoust. Soc. Am. 83, 2322-2327.

Plomp, R., 1994. Noise, amplification, and compression: Considerations of three main issues in hearing aid design. Ear Hear. 15, 2-12.

Plomp, R., Mimpen, A.M., 1979. Improving the reliability of testing the speech reception threshold for sentences. Audiology $18,43-53$.

Ricketts, T., Mueller, H.G., 1999. Making sense of directional microphone hearing aids. Am. J. Audiol. 8, 117-127.

Rosen, S., Fourcin, A., 1986. Frequency selectivity and the perception of speech. In: Moore, B.C.J. (Ed.), Frequency Selectivity in Hearing. Academic, London.

Rosen, S., Faulkner, A., Wilkinson, L., 1999. Adaptation by normal listeners to upward spectral shifts of speech: implications for cochlear implants. J. Acoust. Soc. Am. 106, 3629-3636.

Shannon, R.V., Zeng, F.-G., Kamath, V., Wygonski, J., Ekelid, M., 1995. Speech recognition with primarily temporal cues. Science 270, 303-304.

Shannon, R.V., Zeng, F.-G., Wygonski, J., 1998. Speech recognition with altered spectral distribution of envelope cues. J. Acoust. Soc. Am. 104, 2467-2476.

Simpson, A.M., Moore, B.C.J., Glasberg, B.R., 1990. Spectral enhancement to improve the intelligibility of speech in noise 
for hearing-impaired listeners. Acta Otolaryngol. Suppl. 469, 101-107.

Smoorenburg, G.F., 1992. Speech reception in quiet and in noisy conditions by individuals with noise-induced hearing loss in relation to their tone audiogram. J. Acoust. Soc. Am. 91, 421-437.

Soede, W., Bilsen, F.A., Berkhout, A.J., 1993. Assessment of a directional microphone array for hearing-impaired listeners. J. Acoust. Soc. Am. 94, 799-808.

Srulovicz, P., Goldstein, J.L., 1983. A central spectrum model: a synthesis of auditory-nerve timing and place cues in monaural communication of frequency spectrum. J. Acoust. Soc. Am. 73, 1266-1276.

Steinberg, J.C., Gardner, M.B., 1937. The dependency of hearing impairment on sound intensity. J. Acoust. Soc. Am. 9, 11-23.

Stone, M.A., Moore, B.C.J., 1992. Syllabic compression: Effective compression ratios for signals modulated at different rates. Br. J. Audiol. 26, 351-361.

Stone, M.A., Moore, B.C.J., Alcántara, J.I., Glasberg, B.R., 1999. Comparison of different forms of compression using wearable digital hearing aids. J. Acoust. Soc. Am. 106, 3603-3619.

ter Keurs, M., Festen, J.M., Plomp, R., 1992. Effect of spectral envelope smearing on speech reception. I. J. Acoust. Soc. Am. 91, 2872-2880.

ter Keurs, M., Festen, J.M., Plomp, R., 1993. Effect of spectral envelope smearing on speech reception. II. J. Acoust. Soc. Am. 93, 1547-1552.

Thornton, A.R., Abbas, P.J., 1980. Low-frequency hearing loss: perception of filtered speech, psychophysical tuning curves, and masking. J. Acoust. Soc. Am. 67, 638-643.
Turner, C.W., Burns, E.M., Nelson, D.A., 1983. Pure tone pitch perception and low-frequency hearing loss. J. Acoust. Soc. Am. 73, 966-975.

Tyler, R.S., 1986. Frequency resolution in hearing-impaired listeners. In: Moore, B.C.J. (Ed.), Frequency Selectivity in Hearing. Academic Press, London.

Tyler, R.S., Kuk, F.K., 1990. Consonant recognition and quality judgments of noise-reduction hearing aids. Acta Otolaryngol. Suppl. 469, 224-229.

van Buuren, R.A., Festen, J., Houtgast, T., 1999. Compression and expansion of the temporal envelope: Evaluation of speech intelligibility and sound quality. J. Acoust. Soc. Am. 105, 2903-2913.

Vickers, D.A., Moore, B.C.J., Baer, T., 2001. Effects of lowpass filtering on the intelligibility of speech in quiet for people with and without dead regions at high frequencies. J. Acoust. Soc. Am. 110, 1164-1175.

Villchur, E., 1973. Signal processing to improve speech intelligibility in perceptive deafness. J. Acoust. Soc. Am. 53, 1646-1657.

Young, E.D., Sachs, M.B., 1979. Representation of steady-state vowels in the temporal aspects of the discharge patterns of populations of auditory-nerve fibres. J. Acoust. Soc. Am. 66, 1381-1403.

Yund, E.W., Buckles, K.M., 1995a. Enhanced speech perception at low signal-to-noise ratios with multichannel compression hearing aids. J. Acoust. Soc. Am. 97, 1224 1240.

Yund, E.W., Buckles, K.M., 1995b. Multichannel compression hearing aids: Effect of number of channels on speech discrimination in noise. J. Acoust. Soc. Am. 97, 12061223. 